\title{
$\mathrm{SCIDOC}$ \\ Publishers
}

International Journal of Clinical Dermatology \& Research (IJCDR)

ISSN 2332-2977

\section{Spectacular Improvement of Neumann Pemphigus Vegetans, Treated with Erythromycin: Report of a Case}

Case Report

CH Saadani*, S Gallouj, FZ Mernissi

CHU Hassan II, Department of Dermatology, Morocco.

\section{Abstract}

Pemphigus vegetans is a rare form of pemphigus vulgaris. Its characterized by hypertrophic and vegetating lesions, which typically affects the big folds. We report a case of Neumann pemphigus with involvement of the oral mucosa, with which a spectacular improvement to erythromycin.

Keywords: Pemphigus Vegetans; Neumann Pemphigus; Erythromycin.

Abbreviations: P Veg: Pemphigus Vegetans; PV: Pemphigus Vulgaris.

\section{Introduction}

Pemphigus vulgaris is a rare autoimmune blistering disease, the vegetative form is an exceptional form. It is manifested by vegetating lesions in relief, sitting mainly in the folds but may be of interest to other sites. We report a case of pemphigus vegetans, taking a geographical aspect with involvement of the oral mucosa, With a spectacular response to erythromycin.

\section{Case Synopsis}

A 35 year-old man, followed-up for tuberculous lymphadenitis under treatment by antibacillar drugs, consulted with a three month history of vegetating lesions of the umbilicus, abdomen, thigh and inguinal folds.

The patient reported that four months ago, he presented a painful, hemorrhagic, purulent and erosive lesions, of the oral mucosa, associated with an itchy vesicular-bullous lesions, which evolved towards hypertrophic lesions, of the entire body.

Examination found multiples plaques, which were vegetating, papillomatous, smelly, well limited, with irregularly shaped, (i.e. Figure 1) involving the umbilical site realizing an omphalitis (i.e, Figure 1a, b), abdominal area (i.e, Figure 1c) the right thigh and inguinal folds (i.e, Figure 1d). An erythematous erosive plaques with polycyclic margins were also observed at the upper and lower limbs (i.e. Figure 2), with some pigmented scars on the back. It also noted the presence of hypertrophic verrucous lesions in the angles of mouth, with a fissured and erosive tongue (i.e. Figure 3). The scalp was involved with an erythematous and squamous patches.

The rest of the examination revealed cervical lymphadenopathy with Alteration of the general state.

The skin biopsy showed papillomatous, verrucous epidermal hyperplasia with a suprabasal acantholysis. The dermis is edematous, characterized by intense mixed inflammatory infiltrate consisting of polymorphonuclear leukocytes, eosinophils, lymphocytes and plasma cells.

The Direct immunofluorescence showed a fishnet pattern of deposition of IgG and C3, and indirect immunofluorescence revealed the presence of positive anti-intercellular substance antibodies to $640 \mathrm{IU} / \mathrm{ml}$.

Furthermore, hepatic serology B was positive, contraindicate oral corticosteroids. The patient was then placed under Erythromycin $1 \mathrm{~g} /$ day for a $45 \mathrm{~kg}$ weight, combined with topical treatment with

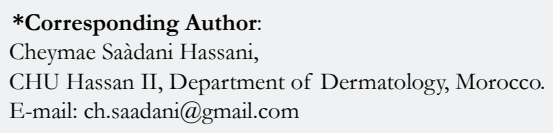

Citation: CH Saadani, S Gallouj, FZ Mernissi. Spectacular Improvement of Neumann Pemphigus Vegetans, Treated with Erythromycin: Report of a Case. Int J Clin Dermatol Res. 2018;6(8):193-196. doi: http://dx.doi.org/10.19070/2332-2977-1800046

Copyright: $\mathbf{C H}$ Saadani ${ }^{\circ}$ 2018. This is an open-access article distributed under the terms of the Creative Commons Attribution License, which permits unrestricted use, distribution and reproduction in any medium, provided the original author and source are credited. 
Figure 1. Multiples plaques, vegetating, papillomatous and smelly.

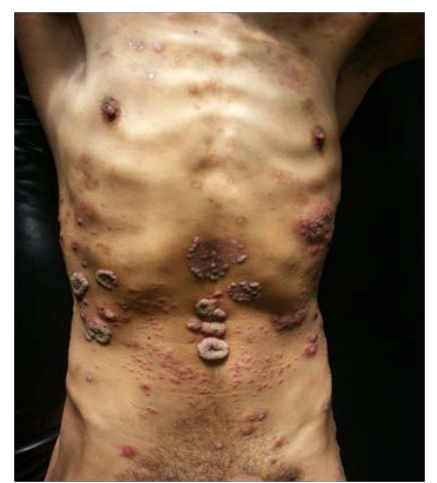

Figure 1a. Vegetating plaques involving the umbilical site.

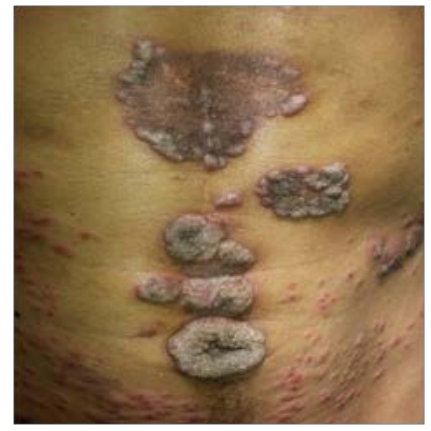

Figure 1b. Omphalitis.

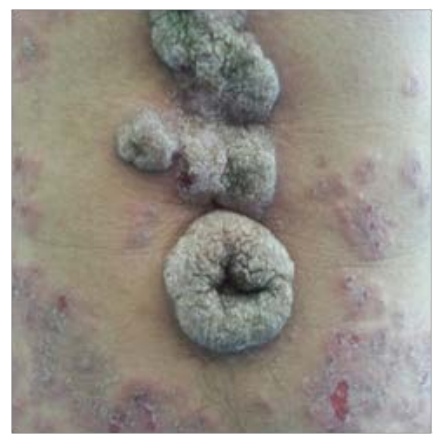

Figure 1c. Vegetating plaques involving the abdominal area

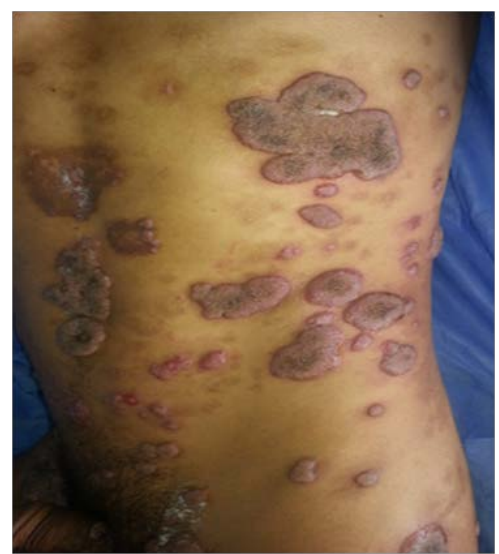

Figure 1d. Vegetating plaques involving the right thigh and inguinal folds.

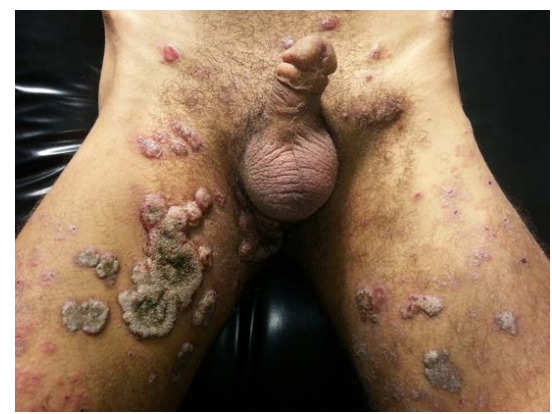

Figure 2. An Erythematous erosive plaques with polycyclic margins.

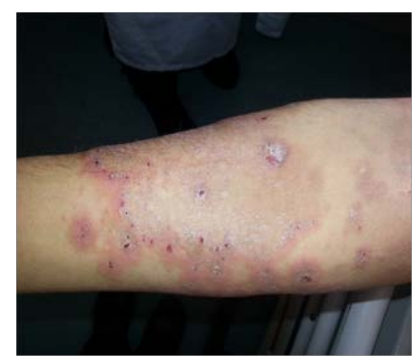

Figure 3. Fissured and erosive tongue.

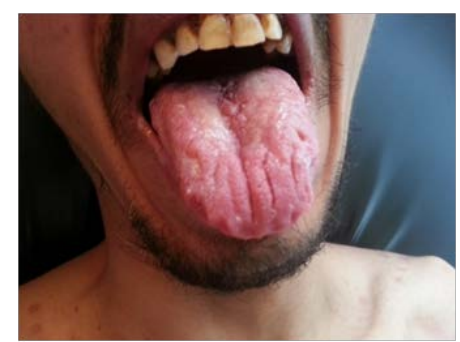


dermocorticoids, antiseptic and wound care.

The evolution was marked by subsidence of lesions in the first week of treatment with no new lesions. The treatment was continued for 3 months. Erythromycin side effects were minor, such as stomach pain managed by omeprazole drugs. The patient is in complete remission for 3 years without relapse (i.e. Figure 4).

\section{Case Discussion}

The term pemphigus refers to a group of autoimmune bullous diseases of the skin and mucous membranes that have variable clinical and prognostic features [1].

Figure 4. The patient is in complete remission.
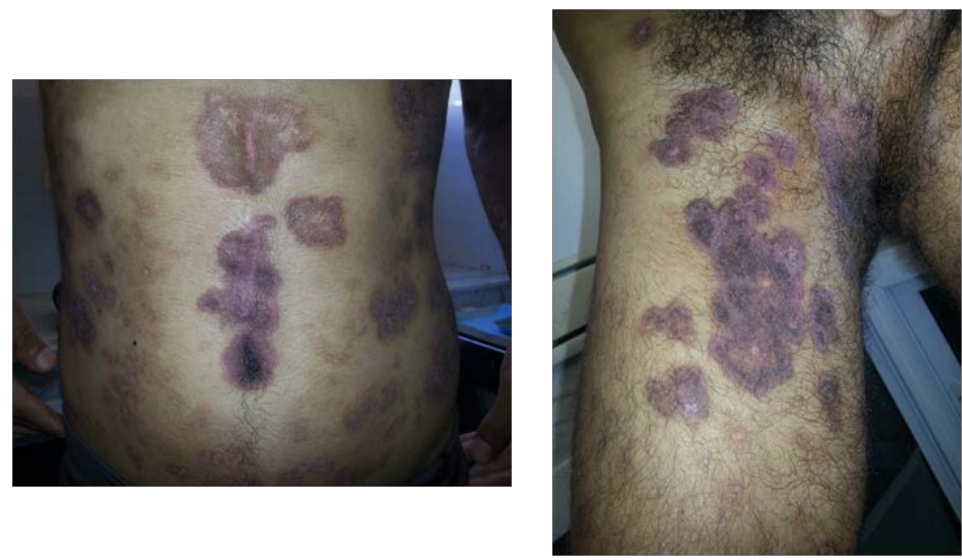

Pemphigus Vegetans (P Veg), which represents only 1-2\% of all pemphigus patients, is thought to be a rare variant of Pemphigus Vulgaris (PV), characterized by vegetating erosions, primarily in flexures. Classically, two subtypes of Pemphigus Vegetans are recognized $[1,7]$ :

- Neumann's subtype: Actually beginning as PV, with vescicles and bullae that rupture to form hypertrophic granulating erosions, then evolving into vegetating exuding masses; which bleed easily [7]. It's the case of our patient.

- Hallopeau's subtype: Initially characterized by pustular lesions that, after rupturing, merge and gradually evolve into vegetating erosions with a centrifugal expansion [7].

The sites most frequently involved are the big folds (axillary, inframammary, inguinocrural, intergluteal), but sometimes areas with small folds are also involved (nasolabial folds, labial folds, periumbelical area) [1]. However, vegetations may occur at any site and not solely in intertriginous areas. In non-flexural sites the granulation tissue can dry out and then produce a warty surface with painful fissures.

Oral involvement is exceedingly common $(60 \%)$ often since the beginning of the disease $(75 \%)$, leading sometimes to malnutrition $[3,7]$. Oral vegetations are rare, especially in the Neumann form, but occasionally the tongue develops a 'cerebriform' appearance which is typical of this condition [2].

The histological features of pemphigus vegetans are a suprabasal acantholysis, an exuberant proliferation of involved epidermis, which shows papillomatous, verrucous or pseudoepitheliomatous hyperplasia. Intraepidermal microabscesses consisting of eosinophils and neutrophils, sometimes interspersed with apoptotic or necrotic keratinocytes, are often seen. They can also invade the hair follicle or sweat duct epithelium.
The diagnosis of $\mathrm{P}$ Veg is based on clinical manifestations, and histology.

Immunofluorescence findings in $\mathrm{P}$ veg are indistinguishable from those of PV. Direct immunofluorescence demonstrates deposition of $\mathrm{IgG}$ and $\mathrm{C} 3$ on the cell surface of keratinocytes. Indirect immunofluorescence reveals circulating antiepithelial cell-surface $\mathrm{IgG}$ as in the reported case.

The physiopathology of the vegetation character of the lesions is not completely elucidated. It would involve local factors (occlusion, maceration and bacterial superinfection at the level of the folds), and general factors. Pemphigus has a genetic basis, characterized by the production of pathogenic autoantibodies directed against different desmosomal proteins (desmoglein 3 and sometimes desmoglein 1 as well) which gives rise to suprabasal acantholysis in both skin and mucous membranes. Some studies have suggested that in P Veg there are additional, occasionally detected antibodies, which bind to other desmosomal proteins, such as desmocollins 1 and 2, and periplakin. This could be a possible explanation for the differences in clinical appearance between PV and P Veg [3].

Under discussion are also immunopathological factors such as Th-2-mediated immune reaction and cytokines that play a role in the epithelial proliferation and eosinophils chemotaxis. Then, activation of the complement cascade in skin lesions attracts inflammatory cells, including polymorphonuclear leukocytes, which cause a severe inflammatory response, with the formation of intraepidermal abscesses and vegetative lesions, both characteristic of P Veg $[2,3]$.

Treatment of P Veg is similar to that for PV, being systemic glucocorticoids the first choice therapy. The Neumann's subtype is more resistant than Hallopeau's subtype, and requires high doses of corticosteroids with sometimes combination with other treatments such as dapsone or other immunosuppressive drugs [2]. 
In response to the infectious contraindication of the patient (Hepatitis $\mathrm{B}$ ), erythromycin was a alternative therapeutic regimens.

Indeed, in addition to the bacteriostatic effect, erythromycin exerts an anti-inflammatory effect, decreasing the production of pro-inflammatory cytokines. This molecule also inhibits neutrophil chemotaxis, lymphocyte proliferation, and eosinophil activity, as well as modulation of the expression of genes involved in the immune response and inflammation $[4,5]$.

Considering all these properties, erythromycin has been used in other chronic inflammatory dermatoses, reported by various studies, such as bullous pemphigoid, linear IgA dermatosis, and atopic dermatitis, administered as monotherapy or in combination With topical steroids, indicated in patients which the systemic corticosteroid therapy is contraindicated, thus sparing the side effects of long-term oral corticosteroids [6].

\section{Conclusion}

Erythromycin has proven effective in pemphigus vegetating, and therefore a good therapeutic alternative to corticosteroids.

\section{References}

[1]. Rmili M, Adamski H, Lopez L, Le FG, Chevrant-Breton J. Pemphigus vegetans: A rare form of pemphigus. Ann Dermatol Venereol. 2011;138(4):3646. doi: 10.1016/j.annder.2010.11.005. PubMed PMID: 21497269.

[2]. Dahbi N, Hocar O, Akhdari N, Amal S, Fakhri A, Rais H, et al. Pemphigus vegetans: rare form of pemphigus. Presse Med. 2014 May;43(5):619-21. doi: 10.1016/j.lpm.2013.11.012. PubMed PMID: 24613065.

[3]. Liegeon AL, Fougerousse AC, Valois A, Bensa Q, Veran Y. Pemphigus vegetants. Presse Med. 2013 Dec;42(12):1669-70. doi: 10.1016/j. lpm.2013.01.062. PubMed PMID: 23688706

[4]. Kwiatkowska B, Maślińska M. Macrolide therapy in chronic inflammatory diseases. Mediators Inflamm. 2012;2012:636157. doi: 10.1155/2012/636157. PubMed PMID: 22969171.

[5]. Labro MT. Immunomodulation mediated by antibacterial agents. Resuscitation. 2006 Aug 1;15(4):259-64.

[6]. Fox BJ, Odom RB, Findlay RF. Erythromycin therapy in bullous pemphigoid: possible anti-inflammatory effects. J Am Acad Dermatol. 1982 Oct;7(4):504-10. PubMed PMID: 6754772.

[7]. Ruocco V, Ruocco E, Caccavale S, Gambardella A, Schiavo AL. Pemphigus vegetans of the folds (intertriginous areas). Clin Dermatol. 2015 JulAug;33(4):471-6. doi: 10.1016/j.clindermatol.2015.04.011. PubMed PMID: 26051064. 